Proceedings volume 10858, Ophtalmic technologies XXIX, 1085813 (2019) ; Proceedings of SPIE BiOS, 2-7 February 2019, San Francisco, California, United States, which should be cited to refer to this work.

DOI: https://doi.org/10.1117/12.2511654

\title{
Device for silent substitution excitation of melanopsin for human eye
}

\author{
Sergiu Agrici ${ }^{1,2}$, Frederic Truffer ${ }^{2}$, Chirojean Balachandran ${ }^{3}$, Aki Kawasaki ${ }^{4}$, and Martial \\ Geiser $^{2}$ \\ ${ }^{1}$ University of Modena and Reggio Emilia \\ ${ }^{2}$ University of Applied Sciences of western Switzerland, Sion, Switzerland \\ ${ }^{3}$ ENSEA, Cergy, France \\ ${ }^{4}$ Hopital Jules Gonin, Lausanne, Switzerland \\ *Corresponding author: martial.geiser@hevs.ch
}

\begin{abstract}
Research in neurobiology has identified a new ocular photoreceptor (melanopsin or ipRGC) which mediates a variety of light-based, non-visual effects on human physiology. One way to isolate the stimulation of ipRGCs is the silent substitution technique. We have built a Maxwellian view device capable of $85 \%$ ipRGCs contrast excitation with a large FOV $\left(52^{\circ}\right)$. Four modulated LED light sources, illuminate a diffusing sphere, which exit aperture is imaged into the pupil of the eye. A camera with a $900 \mathrm{~nm}$ illumination capture the pupil.

Without luminance changes $\left(510 \pm 2 \mathrm{~lm} / \mathrm{m}^{2}\right)$, we increased ipRGC excitation from low to high level on three subjects. We observed a pupil constriction increasing with the ipRGC contrast. This suggests that we excite melanopsin silently. However, further experiments with electrophysiological and pupil recording needs to be done to completely validate our silent substitution device.
\end{abstract}

\section{Introduction}

Research in neurobiology has identified a new ocular photoreceptor with a unique photopigment, melanopsin. This photoreceptor is an intrinsically photosensitive retinal ganglion cell (ipRGC) which mediates a variety of light-based, non-visual effects on human physiology depending on the illumination environment. ${ }^{1}$

Isolated stimulation of the ipRGC on humans is difficult due to overlapping spectral sensitivity with other ocular photoreceptors (rods and cones). The silent substitution is a technique that permits the modulation of one photoreceptor type at a time, maintaining the rest at a steady state. By exploiting the metamers properties, it is possible to stimulate the melanopsin photopigment while maintaining a constant level of cone excitation.

\section{Silent substitution technique}

The basis of melanopsin light stimulation is the silent substitution technique ${ }^{2}$ which is linked to black metamers only differentiated by melanopsine photodetectors. Considering that the eye has 5 specific types of photosensitive cells (L, M and S cones, rods and ipRGCs), the light stimulus can be considered as a 5-dimensional vector. Assuming that only the photopic range of illumination activates melanopsin ${ }^{3}$ so that the rods are deactivated, ${ }^{4}$ we can restrict the light stimulus vector to 4-dimension. In order to modulate the excitation of ipRGCs, at least four different light sources with different colors are required. This approach considers that any light stimulus is composed of a fundamental colored stimulus and a metameric black which excites only the melanopsin component of ipRGC photoreceptors.

With $S_{p}(\lambda)$ being the sensitivity of the photoreceptor $p$ and $I_{q}(\lambda)$ the $q^{\text {th }}$ light source intensity, the photoreceptor stimulation $P S_{p q}$ is given by :

$$
P S_{p q}=\sum_{i}\left[S_{p}\left(\lambda_{i}\right) \cdot K_{p}\right] \cdot\left[I_{q}\left(\lambda_{i}\right) \cdot L R_{q}\right]
$$


where $L R_{q}$ is the luminance ratio of the light source $I_{q}(\lambda)$ and $K_{p}$ a factor for converting power into photometric units. $K$ is well defined for the L and M (683) cones, but not for the S cone and ipRGCs, as only L and M contribute to the luminance. ${ }^{5}$ Applying this to all photoreceptors for all illuminations, equation (1) becomes

$$
P S=P 2 C \cdot L R
$$

where $P 2 C$ is the matrix with the elements calculated as follows:

$$
P 2 C_{p q}=\sum_{i} S_{p}\left(\lambda_{i}\right) \cdot I_{q}\left(\lambda_{i}\right)
$$

This means that the 4-dimensional space of the light sources is projected through the $P 2 C$ matrix into the 4 -dimensional space of the photoreceptors. Note that any pair $(P S, L R)$ has a physical meaning only if all components of $L R$ are positive. For the silent substitution, we set the photoreceptor stimulation vector $P S$ and get the luminance ratio vector $L R$ of the light source by the following equation

$$
L R=P 2 C^{-1} \cdot P S
$$

On the basis of these calculations, a sequence of black metamers is produced. This induces a signal whose maximum contrast for ipRGC excitation depends on the spectrum of the light sources.

\section{Existing devices}

Basically, such devices are based on two types of optical arrangements: Maxwellian view in which the pupil of the instrument (which could be an artificial pupil) defines the position of the eye and Newtonian or natural view in which only the eye and its own pupil is used. For Maxwellian view, the object plane is usually at a distance corresponding to the resting position of the lens of the eye. For Newtonian view, the object plane is at least 20 $\mathrm{cm}$ away from the corneal surface of the eye.

\begin{tabular}{lllc} 
Reference & FOV, pattern & $\lambda[\mathrm{nm}]$ & $C$ \\
\hline $\begin{array}{lll}\text { Maxwellian view } \\
6-8\end{array}$ & $30^{\circ}$, c. obsc. $10.5^{\circ}$ & $456,487,540,592,633$ & $17 \%$ \\
9 & $27.5^{\circ}$, c. obsc. $5^{\circ}$ & up to $56 \lambda$ & $50 \%$ \\
\hline Newtonian view & & \\
10 & 2 rings & $468,524,599,633$ & $4 \%$ \\
5 & $20^{\circ}$, homegeneous & $470,525,500,670$ & $53 \%$ \\
11 & homogeneous & $466,514,590,634$ & - \\
12 & $18.9^{\circ}$, homogeneous & $468,507,593,633$ & $53 \%$ \\
13 & $95^{\circ}$ & $470,510,595,635$ & $11 \%$ \\
14 & $28.1^{\circ}$ & $468,508,593,633$ & $20 \%$ \\
15 & $25^{\circ} \times 12^{\circ}$ & $465,500,515,595,635$ & - \\
16 & $1^{\circ}$ or $20^{\circ}$ homogeneous & $447,470,505,530,590,627$ & $19 \%$ \\
17 & $19^{\circ}$, homogeneous & $460,525,635,445 / 555$ & $21 \%$ \\
18 & homogeneous & $447,472,502,523,594,637,656$ &
\end{tabular}

Table 1. Summary of the main instrumentation reference values for the excitation of ipRGC photoreceptors. FOV is the field of view and $C$ the maximal ipRGC contrast which can be produced.

We found 14 articles (see table 1) in the peer-review scientific literature since 2010 dealing with silent substitution and melanopsin excitation. We also found more articles which are not aimed at melanopsin ganglion cell excitation but described interesting instrumentation for silent substitution technique, one describing a true retinal projection. ${ }^{19}$

Levels of illumination are not easy to compare because scholars report either lumen per square centimeter $\left(\mathrm{lm} / \mathrm{cm}^{2}\right)$ or troland $(\mathrm{Td})$, but do not give the necessary information to convert one to the other. Given values are either between 300 to $2500 \mathrm{~lm} / \mathrm{cm}^{2}$ or between 300 to $30000 \mathrm{Td}$ but mostly around $3000 \mathrm{Td}$. Light stimuli 
are usually presented homogeneously over a given area of the retina, either in an annulus or in a given circle centered on the fovea or outside of the fovea. ${ }^{5-18}$

For silent substitution devices, instruments with Newtonian view are more common and are used in a larger number of studies than those with Maxwellian view. For the latter, out of five publications, three are based on the same instrumentation. ${ }^{6-8}$ Another group of four publications proposes custom-built instruments accompanied by very vague explanation regarding the construction and working principle. ${ }^{15,16,18}$ Spitschan $^{9}$ demonstrates $50 \%$ of contrast with a Maxwellian setup by using an artificial pupil and a digital spectral modulator. Only one commercially available solution for natural view could potentially be used for ipRGC excitation (ColorDome,Diagnosys,Cambridge,UK) because it includes 4 different light sources but are aimed at color stimulation only. ${ }^{11,20,21}$

\section{Material and methods}

\section{Instrumentation}

We propose a new device with a homogeneous $52^{\circ}$ field of view (FOV) based on a Maxwellian view able to achieve a high contrast (theoretically 85\%) for ipRGC excitation. The exit pupil diameter of the device was set to $2.5 \mathrm{~mm}$ but can easily be reduced to $1 \mathrm{~mm}$, which requires a subject's pupil slightly larger than this value to accept slight lateral movements. In our system, the pupil diameter of the eye under light excitation is measured and not the contralateral eye which would be required if an artificial pupil is used.

Figure 1. System layout with the optical parts, the light sources and the control system.

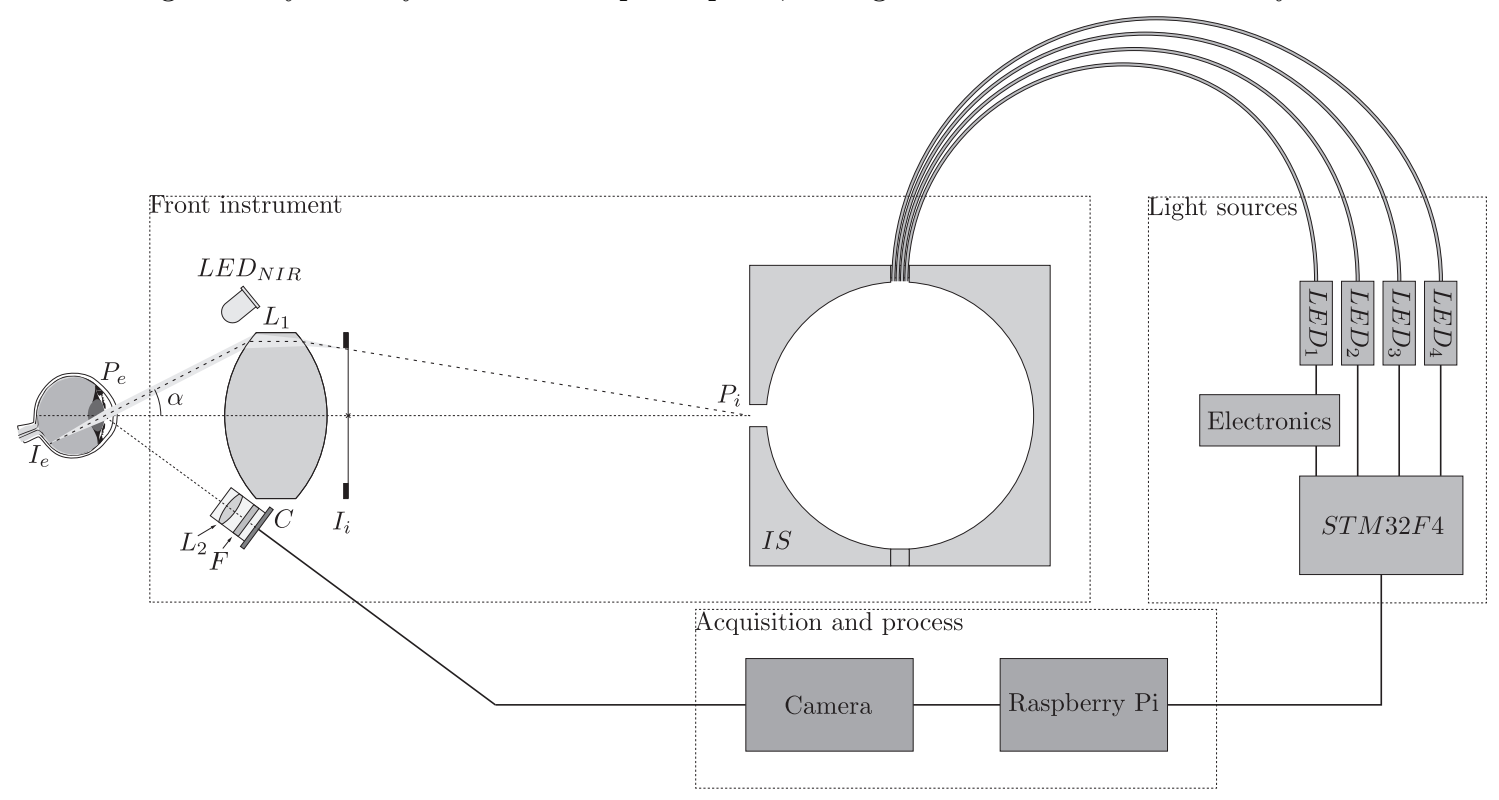

The four light sources: $L E D_{1}$ (A42182, Seoul Semiconductor), $L E D_{2}, L E D_{3}$ and $L E D_{4}$ (M405F1,M505F1 and M625F2, ThorLabs) modulated with pulse width modulation signal, are homogenized through an integrating sphere $I S$. The LEDs are controlled with a microprocessor (STM32F4). The exit port $P_{i}$ of the sphere is imaged onto the pupil of the eye $P_{e}$ with lens $L_{1}$ with a 5:2 magnification. The surface $I_{i}$, with a central dot for eye fixation purpose, is projected onto the retina of the subject. Four NIR led at $900 \mathrm{~nm}$ illuminate the pupil which is observed with a camera fixed below the lens $L_{1}$. The camera includes a lens $L_{2}$ and a high pass filter $F$ to avoid visible light from the integrating sphere. During light experiments, a video at 24 frames per second is acquired. The red channel is processed on a Raspberry Pi with the library opencv in python. A Gaussian filter is applied in order to remove noise and each pixel are then reduced to one bit. Pupil contour is extracted and an ellipse is fitted to this contour which gives the size and the position of the pupil. If no contour is found, image is considered as part of eye blinking. 


\section{Luminance}

The luminance $L_{v}$ is the result of $\mathrm{L}$ and $\mathrm{M}$ cones excitation. In case that the cones are silently stimulated, then the luminance remains constant. Its was computed as following

$$
L_{v}=\sum_{\lambda}\left(\frac{I_{555} \cdot S(\lambda) \cdot V(\lambda)}{R(\lambda) \cdot \Omega \cdot A} \cdot 683\right)\left[\frac{\mathrm{cd}}{\mathrm{m}^{2}}\right]
$$

where $I_{555}$ is the optical power (measured with the powermeter PM100D, ThorLabs, set at $\left.555 \mathrm{~nm}\right), S(\lambda)$ the power spectrum, $V(\lambda)$ the light efficiency function of the eye normalized to $683, R(\lambda)$ the responsiveness function of the powermeter, $\Omega$ the solid angle sustended by the illumination $\left(\Omega=0.636\right.$ for a FOV of $\left.\pm 26^{\circ}\right)$ and $A$ the area of the exit pupil of the instrument with $2.5 \mathrm{~mm}$ in diameter.

Figure 2. Illumination spectrum for the low and high level of ipRGC excitation with identical luminance and color.

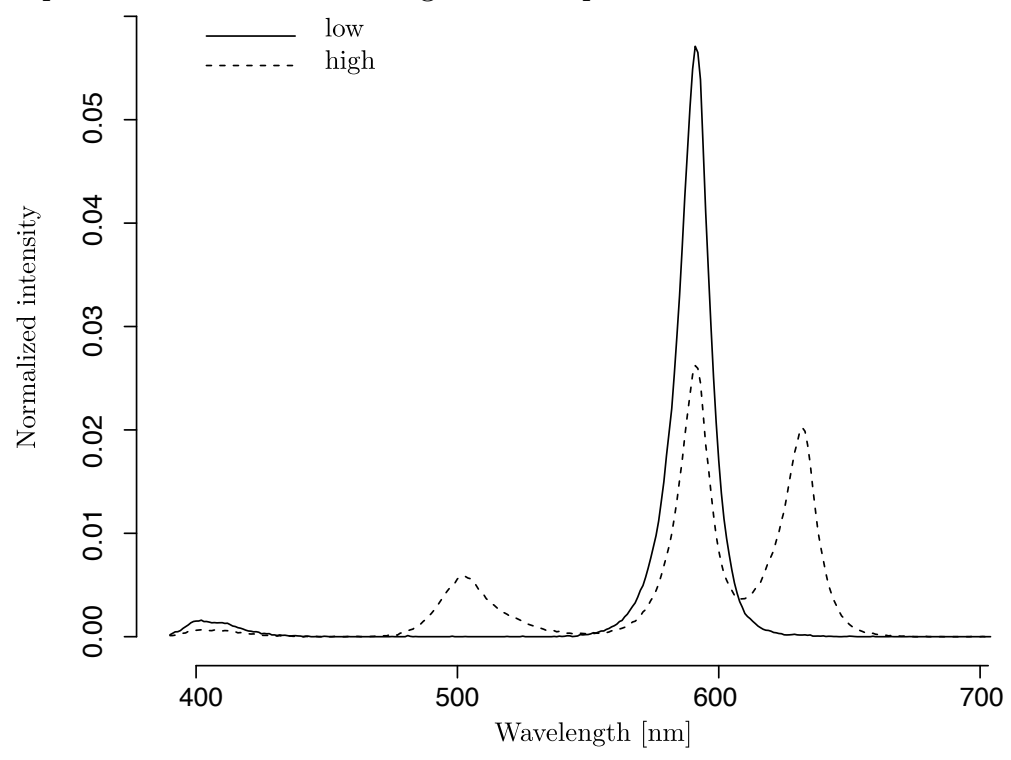

The calculated luminance $L_{v}$ for different excitation levels of ipRGCs varies little $( \pm 0.44 \%)$ up to a contrast level $C$ of $70 \%$. Table 2 shows the measurement of $L_{v}$ at the contrast levels used for the evaluation of the instrument.

$$
\begin{array}{lccccc}
\text { Contrast } C[\%] & 0 & 20 & 50 & 70 & \text { from } 0 \text { to } 70 \\
L_{v}\left[\mathrm{~cd} / \mathrm{m}^{2}\right] & 511.4 & 511.6 & 509.6 & 506.7 & 509.8 \pm 2.3
\end{array}
$$

Table 2. Measured $L_{v}$ values for different ipRCGs contrast.

Optical validations were performed by measuring the spectrum variation for different excitation steps (see example in Figure 2).

\section{Experiments}

We performed two experiments:

1. L-cone: We exposed three eyes to a bluish light background $\left(50 \mathrm{~cd} / \mathrm{m}^{2}\right)$. At 7 -second intervals, we added, during one second, red light with logarithmic increasing luminosity $L_{v}$ from $1 \mathrm{~cd} / \mathrm{m}^{2}$ to $316 \mathrm{~cd} / \mathrm{m}^{2}$. 
2. ipRGC: We exposed 3 eyes to a contrast change of resp. 20\%, $50 \%$ and $70 \%$ at a constant luminosity of $510 \mathrm{~cd} / \mathrm{m}^{2}$ in the following order (see upper-left figure 4): 12s baseline at $P S=(S, L, M$, ipRGC) $=$ $(28,224,137,12), 12 \mathrm{~s}$ at resp. $i p R G C=18,36$ and 57 then back to baseline value. Duration of the transition was $0.5 \mathrm{~s}$ and change was linear on $i p R G C$. We did not record the pupil diameter from darkness to light and from light to darkness before and after the measurement (no long recovery).

\section{Results}

1. L-cone: As shown in the literature ${ }^{22}$ we found a linear relationship between the normalized relative constriction of the pupil $n C_{p}$ and luminance of the red excitation $L_{v}, n C_{p}=0.5-12.5 \cdot \log \left(L_{v}\right), R^{2}=0.9758$ (figure 3).

2. ipRGC: For all subjects, the pupil diameter was $4.55 \pm 1.63 \mathrm{~mm}$ and the minimum was $2.97 \mathrm{~mm}$ which is above the exit pupil diameter of our experimental set-up. The changes of the pupil diameter increase with the increase of the contrast of the ipRGC excitation (see figure 4 right).

Figure 3. Experiment a) L-cone. Mean results for the L cone excitation. Upper-right: one single recording at $\log \left(L_{v}\right)=2.5$.

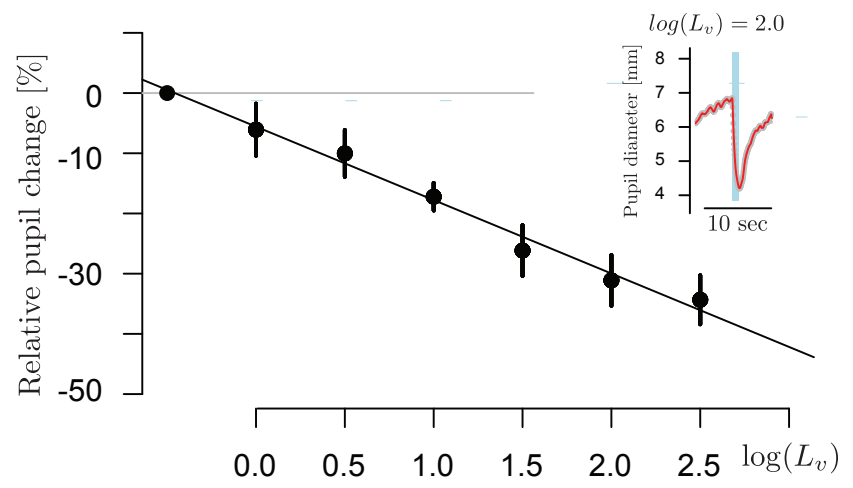

Figure 4. Experiment b) ipRGC. Top-left: time change of the ipRGC excitation. Bottom-left: example of the mean of normalized pupil diameter of 5 recordings on one subject at $70 \%$ ipRGC contrast. Right: mean and standard deviation of the pupil diameter change from low to high and from high to low ipRGC excitation for different contrast.

ipRGC excitation level

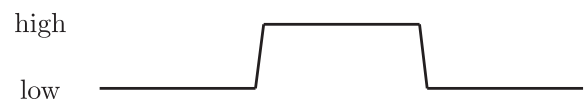

Mean pupil diameter change [\%]
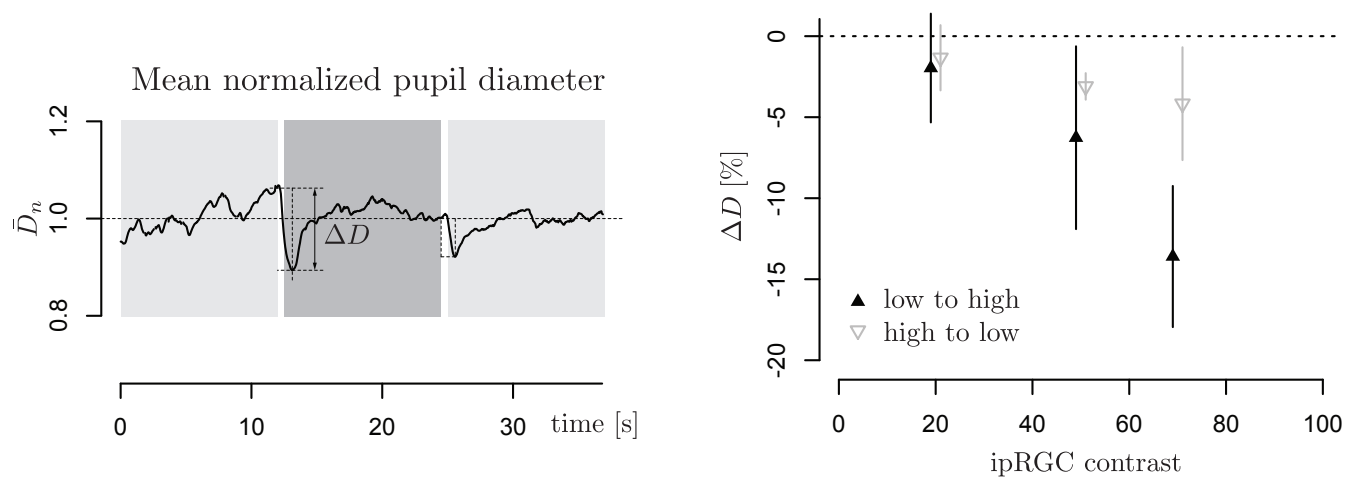

\section{Conclusion}

We demonstrate a working light stimulus device which appear to excite melanopsin ipRGC independently of simultaneous cone excitation. A detailed quantification of the time variation of the pupil waveform parameters 
is the next step and will help to better differentiate cones excitation from ipRGC. Simultaneous psychophysical and electrophysiological recording with the pupil recording will also help to validate the silent substitution. Compared to instrumentation reported in the literature, our device exhibits higher FOV and potentially higher ipRGC contrast. The optical system has a precise exit pupil where the eye's pupil is located. The instrument can be aligned in the same way as a fundus camera.

\section{Thanks}

We are grateful to Prof. Sei-ichi Tsujimura for the many helpful and constructive discussions.

\section{REFERENCES}

[1] Münch, M. and Kawasaki, A., "Intrinsically photosensitive retinal ganglion cells: classification, function and clinical implications," Current Opinion in Neurology 26, 45-51 (Feb. 2013).

[2] Estvez, O. and Spekreijse, H., "The silent substitution method in visual research," Vision Research 22, 681-691 (Jan. 1982).

[3] Lucas, R. J., Hattar, S., Takao, M., Berson, D. M., Foster, R. G., and Yau, K.-W., "Diminished Pupillary Light Reflex at High Irradiances in Melanopsin-Knockout Mice," Science 299, 245-247 (Jan. 2003).

[4] Aguilar, M. and Stiles, W. S., "Saturation of the Rod Mechanism of the Retina at High Levels of Stimulation," Optica Acta: International Journal of Optics 1, 59-65 (Jan. 1954).

[5] Tsujimura, S.-i., Ukai, K., Ohama, D., Nuruki, A., and Yunokuchi, K., "Contribution of human melanopsin retinal ganglion cells to steady-state pupil responses," Proceedings of the Royal Society B: Biological Sciences 277, 2485-2492 (Aug. 2010).

[6] Cao, D., Nicandro, N., and Barrionuevo, P. A., "A five-primary photostimulator suitable for studying intrinsically photosensitive retinal ganglion cell functions in humans," Journal of Vision 15, 15.1.27 (Jan. 2015).

[7] Barrionuevo, P. A. and Cao, D., "Luminance and chromatic signals interact differently with melanopsin activation to control the pupil light response," Journal of Vision 16 (Sept. 2016).

[8] Zele, A. J., Feigl, B., Adhikari, P., Maynard, M. L., and Cao, D., "Melanopsin photoreception contributes to human visual detection, temporal and colour processing," Scientific Reports 8 (Mar. 2018).

[9] Spitschan, M., Jain, S., Brainard, D. H., and Aguirre, G. K., "Opponent melanopsin and S-cone signals in the human pupillary light response," Proceedings of the National Academy of Sciences 111, 15568-15572 (Oct. 2014).

[10] Tsujimura, S.-i. and Tokuda, Y., "Delayed response of human melanopsin retinal ganglion cells on the pupillary light reflex," Ophthalmic 8 Physiological Optics: The Journal of the British College of Ophthalmic Opticians (Optometrists) 31, 469-479 (Sept. 2011).

[11] Barrionuevo, P. A., Nicandro, N., McAnany, J. J., Zele, A. J., Gamlin, P., and Cao, D., "Assessing Rod, Cone, and Melanopsin Contributions to Human Pupil Flicker Responses," Investigative Ophthalmology $\&$ Visual Science 55, 719-727 (Feb. 2014).

[12] Fukuda, Y., Tsujimura, S.-i., Higuchi, S., Yasukouchi, A., and Morita, T., "The ERG responses to light stimuli of melanopsin-expressing retinal ganglion cells that are independent of rods and cones," Neuroscience Letters 479, 282-286 (Aug. 2010).

[13] Brown, T. M., Tsujimura, S.-i., Allen, A. E., Wynne, J., Bedford, R., Vickery, G., Vugler, A., and Lucas, R. J., "Melanopsin-based brightness discrimination in mice and humans," Current biology: CB 22, 11341141 (June 2012).

[14] Fukuda, Y., Higuchi, S., Yasukouchi, A., and Morita, T., "Distinct responses of cones and melanopsinexpressing retinal ganglion cells in the human electroretinogram," Journal of Physiological Anthropology 31, 20 (June 2012).

[15] Woelders, T., Leenheers, T., Gordijn, M. C. M., Hut, R. A., Beersma, D. G. M., and Wams, E. J., "Melanopsin- and L-coneinduced pupil constriction is inhibited by S- and M-cones in humans," Proceedings of the National Academy of Sciences of the United States of America 115, 792-797 (Jan. 2018).

[16] Horiguchi, H., Winawer, J., Dougherty, R. F., and Wandell, B. A., "Human trichromacy revisited," Proceedings of the National Academy of Sciences 110, E260-E269 (Jan. 2013). 
[17] Fukuda, Y., Kai, A., Tsujimura, S.-i., Higuchi, S., and Morita, T., "Human Melatonin Suppression in Response to Silent Substitution Stimuli of Photoreceptors," Journal of Science and Technology in Lighting 41, 211-216 (Mar. 2018).

[18] Vinot, F., Brettel, H., Dang, T.-V., and Rohellec, J. L., "Domain of metamers exciting intrinsically photosensitive retinal ganglion cells (ipRGCs) and rods," JOSA A 29, A366-A376 (Feb. 2012).

[19] Klee, S., Link, D., Bessler, P., and Haueisen, J., "Optoelectrophysiological stimulation of the human eye using fundus-controlled silent substitution technique," Journal of Biomedical Optics 16, 015002 (Jan. 2011 ).

[20] Maguire, J., Parry, N. R. A., Kremers, J., Kommanapalli, D., Murray, I. J., and McKeefry, D. J., "Rod Electroretinograms Elicited by Silent Substitution Stimuli from the Light-Adapted Human Eye," Translational Vision Science \& Technology 5, 13-13 (July 2016).

[21] Maguire, J., Parry, N. R. A., Kremers, J., Murray, I. J., and McKeefry, D., "The morphology of human rod ERGs obtained by silent substitution stimulation," Documenta Ophthalmologica 134, 11-24 (Feb. 2017).

[22] Kostic, C., Crippa, S. V., Martin, C., Kardon, R. H., Biel, M., Arsenijevic, Y., and Kawasaki, A., "Determination of Rod and Cone Influence to the Early and Late Dynamic of the Pupillary Light Response," Investigative Ophthalmology \& Visual Science 57(6), 2501-2508 (2016). 